\title{
Terrestrial Planet Finder: science overview
}

\section{Stephen C. Unwin, Charles A. Beichman}

Stephen C. Unwin, Charles A. Beichman, "Terrestrial Planet Finder: science overview," Proc. SPIE 5487, Optical, Infrared, and Millimeter Space Telescopes, (12 October 2004); doi: 10.1117/12.550949

SPIE Event: SPIE Astronomical Telescopes + Instrumentation, 2004, Glasgow, United Kingdom 


\title{
Terrestrial Planet Finder: Science Overview
}

\author{
S. C. Unwin ${ }^{\mathrm{a}}$ and C. A. Beichman ${ }^{\mathrm{b}}$ \\ a Jet Propulsion Laboratory, California Institute of Technology, Mail Stop 301-486, \\ 4800 Oak Grove Drive, Pasadena, CA 91109-8099, USA \\ ${ }^{\mathrm{b}}$ Michelson Science Center, California Institute of Technology, Mail Code 100-22, \\ 770 South Wilson Avenue, Pasadena, CA 91125, USA
}

\begin{abstract}
The Terrestrial Planet Finder (TPF) seeks to revolutionize our understanding of humanity's place in the universe - by searching for Earth-like planets using reflected light, or thermal emission in the mid-infrared. Direct detection implies that TPF must separate planet light from glare of the nearby star, a technical challenge which has only in recent years been recognized as surmountable. TPF will obtain a low-resolution spectrum of each planet it detects, providing some of its basic physical characteristics and its main atmospheric constituents, thereby allowing us to assess the likelihood that habitable conditions exist there. NASA has decided the scientific importance of this research is so high that TPF will be pursued as two complementary space observatories: a visible-light coronagraph and a mid-infrared formationflying interferometer. The combination of spectra from both wavebands is much more valuable than either taken separately, and it will allow a much fuller understanding of the wide diversity of planetary atmospheres that may be expected to exist. Measurements across a broad wavelength range will yield not only physical properties such as size and albedo, but will also serve as the foundations of a reliable and robust assessment of habitability and the presence of life.
\end{abstract}

Keywords: Extrasolar planets, coronagraph, interferometer, comparative planetology

\section{INTRODUCTION}

The Terrestrial Planet Finder (TPF) is perhaps the most challenging astronomy mission yet undertaken. It has the very bold objective of detecting Earth-like planets around nearby stars, measuring their physical and chemical characteristics, and, perhaps, finding evidence of life through the presence of spectroscopic 'biomarkers'. As a field of scientific research, the study of extrasolar planets is still in its infancy, but is rapidly growing, as the richness of problems in the astronomy of small bodies become more accessible to observation and modeling.

In this paper, we focus on the scientific drivers for the search for Earth-like planets, and the importance of measuring their spectra as a probe of their physical properties and atmospheric constituents. Other papers in this conference proceedings volume cover many aspects of this work which will lead to launch of the NASA Terrestrial Planet Finder and its European counterpart, Darwin. Key technologies are now sufficiently mature that the design and construction of such an instrument is now possible $\left(\right.$ Laskin $\left.^{1}\right)$. In the optical, we are developing the ability to fabricate large, ultrapolished optics, to correct surface defects with very stable deformable mirrors, and suppress on-axis starlight through innovative techniques involving combinations of masks and stops. In the mid-infrared, the ability to maintain a broadband fringe null is now being demonstrated; by placing the star on-axis at the null position, light from a faint planet in an off-axis position can be detected. Both of these techniques - optical and mid-IR - are being pursued though laboratory testbeds, numerical modeling, and space instrument design. Many challenges are involved in bringing these technologies and engineering challenges to fruition in space missions to detect light from extrasolar planets.

Probably the biggest advance in this field was the first detection of an extrasolar planet, via precision Doppler (radial velocity RV) techniques (Mayor \& Queloz ${ }^{2}$; Marcy \& Butler ${ }^{3}$ ). Since then, over 120 planets have been discovered; most of these planets are Jupiter-like, and most of the systems do not look much like our own Solar System. Even so, 
the impetus to search for Earth-like planets is very great. The RV method cannot detect planets as small as an Earth, but there is every indication from modeling of the stability and evolution of planetary systems (Ida \& $\operatorname{Lin}^{4}$ ) that 'Earths' may in fact be common around nearby stars.

Several other techniques have the promise of further exploring the diversity of planetary systems. Detection of the astrometric 'wobble' signature of the parent star due to the gravitational pull of the planet is important because such measurements yield the mass of the planet - perhaps the single most important parameter for a body. Ground-based interferometers such as the Keck Interferometer (Colavita et al. ${ }^{5}$; van Belle et al. ${ }^{6}$ ) will be able to detect massive planets. The Space Interferometry Mission (SIM) is being designed to survey at least the nearest 200 stars for planets down to a few Earth masses, and to measure their masses $\left(\right.$ Unwin $\left.^{7}\right)$. Microlensing of stars in our Galaxy offers a sensitive, albeit non-repeatable, method of detecting low-mass planets in a statistical sense (Snodgrass, Horne, and Tsapras $^{8}$; Bond et al. ${ }^{9}$ ).

Developing statistics of the properties of planetary systems is important to understanding their diversity and to answering whether our own Solar System is typical or unusual. Current knowledge suggests our system is unusual, but selection effects in the current (RV) statistics prevent any definitive statement. The NASA Kepler mission (Borucki et al. ${ }^{10}$ ) and the European COROT mission will each survey a large sample of stars for transiting planets. Kepler will be sensitive to a wide range of masses and should yield good statistics on planet frequency, orbit radius and mass distributions, etc. Unfortunately, because the Kepler stars are distant, planets it detects will not be candidates for study with TPF. However, the combined knowledge from all of the ground-based observations, modeling of system stability and evolution, and space-based experiments, will provide a target list for TPF, and a good indication of the number of Earths that TPF might detect. The possible habitability of these planets will have to await TPF itself - the mission designed specifically for this task.

TPF will directly detect light from planets by suppressing the nearby starlight. This simply-stated requirement represents a formidable technical challenge. TPF must also take low-resolution spectra of any planets that it detects, as a probe of the atmospheric conditions of the planet. Because the planet is faint (around $\mathrm{V}=30$ in the visible), not only is a large telescope needed, but also great stability over periods up to hours, lest the planet signal be overwhelmed by variable 'leakage' from the bright star. Because the technical challenges are so great, it is easy to come up with science objectives that are well beyond our abilities to achieve in the next decade or so. Therefore, considerable effort has gone into developing a set of goals that are realistic and feasible, but which, if met, fully justify the large commitment of resources and time that are required to deliver our desired scientific results.

An instrument as capable as the Terrestrial Planet Finder will be able to address other astronomical problems besides the direct detection of extrasolar Earth-like planets and spectroscopy of detected planets. Most important will be in the developing field of Comparative Planetology. TPF will detect other constituents of each star that it searches. Multiple-planet systems are already known to be common. TPF will perform a census of planets around each star, over a wide range of planet masses and orbit radii. In addition, zodiacal dust emission - the remnants of the circumstellar disk of dust and gas out of which planets form - will be readily detectable, and in some cases, the brightness of this emission may hinder the detection of Earth-like planets. But the characterization of zodiacal disks, including possible structure within those disks due to gravitational interaction with (possibly unseen) planets, will provide a rich observational database against which theories and models of the formation and evolution of planetary systems can be challenged. Planetary scientists will have a sample of systems around other stars with which to compare our Solar System. TPF will be in a unique position to advance our understanding of planetary systems, not just individual planets.

In addition to detecting and characterizing Earth-like planets, and extrasolar planetary systems, TPF will be capable of a wide range of studies in more general astrophysics. While scientifically important in their own right, these investigations would not be allowed to compromise the primary mission of TPF in any way. We briefly consider some of the exciting general astrophysics opportunities at the end of this paper, in Section 4. 


\section{SCIENCE OBJECTIVES}

The science objectives for TPF described below are derived from the Executive Summary of the TPF Science Objectives - a draft document developed by the TPF Science Working Group to assist the TPF Project in designing instrument and mission capabilities to meet the highest-level goals for the mission. These objectives are based on current knowledge of the field of extrasolar planets, and current understanding of what measurements will be important for TPF to make. Of particular interest is the number of stars that TPF should search. The requirements are stated in terms of the capability to search a well-defined parameter space, rather than in terms of a required yield of planets. This approach is consistent with the setting of requirements for other astronomy missions: while specific scientific goals always drive the objectives, the instrument and mission must be designed to achieve a specific capability.

Because of the importance of obtaining spectra in both the optical and mid-IR wavebands, we defer discussion of the scientific rationale for combining the wavebands to Section 3 below.

\subsection{Objectives for TPF}

The objectives of the Terrestrial Planet Finder (TPF) are to detect directly and characterize Earth-like planets around nearby stars. The ability of direct detection implies that TPF must separate planet light from starlight. The ability to characterize implies that TPF must determine the type of planet and characterize its gross physical properties and its main atmospheric constituents, thereby allowing an assessment of the likelihood that life or habitable conditions exist there.

Types of Stars. On astrophysical grounds, Earth-like planets should be found around stars that are roughly similar to the sun. Therefore TPF target stars should include main sequence F, G, and K stars.

Terrestrial Planets. Considering the radii and albedos or effective temperatures of solar system planets, TPF must be able to detect terrestrial planets different from our own, down to a minimum terrestrial planet defined as having $1 / 2$ Earth surface area and Earth albedo. At visible wavelengths we assume the phase function of a Lambertian sphere. In the infrared, the minimum detectable planet would be one with an infrared emission corresponding to the surface area and optical albedo given above, positioned in the orbital phase space stipulated below.

Habitable Zone: TPF should search the most likely range as well as the complete range of temperatures within which life may be possible on a terrestrial type planet. In the Solar System, the most likely zone is near the present Earth, and the full zone is the range between Venus and Mars. The habitable zone (HZ) is here defined as the range of semi-major axes from 0.7 to $1.5 \mathrm{AU}$ scaled by the square root of stellar luminosity. The minimum terrestrial planet must be detectable at the outer edge of the $\mathrm{HZ}$.

Orbital Phase Space: The distribution of orbital elements of terrestrial type planets is presently unknown, but observations suggest that giant planet orbits are distributed roughly equally in semi-major axis, and in eccentricity up to those of the solar system planets and larger. Therefore TPF must be designed to search for planets drawn from uniform probability distributions in semi-major axis over the range 0.7 to $1.5 \mathrm{AU}$ and in eccentricity over the range 0 to 0.35 , with the orbit pole uniformly distributed over the celestial sphere with random orbit phase.

Giant Planets. The occurrence and properties of giant planets may determine the environments of terrestrial planets. We require the TPF field of view and sensitivity must be sufficient to detect a giant planet with the radius and geometric albedo or effective temperature of Jupiter at $5 \mathrm{AU}$ (scaled by the square root of stellar luminosity) around at least $50 \%$ of its target stars. A signal-to-noise ratio of at least 5 is required.

Exozodiacal Dust. Emission from exozodiacal dust is both a source of noise and a legitimate target of scientific interest. TPF must be able to detect planets in the presence of zodiacal clouds at levels up to a maximum of 10 times 
the brightness of the zodiacal cloud in the solar system. For the average case, we adopt an expected level of zodiacal emission around target stars of 3 times the level in our own solar system and with the same fractional clumpiness as our solar system's cloud. From a science standpoint, determining and understanding the properties of the zodiacal cloud is essential to understanding the formation, evolution, and habitability of planetary systems. Thus, TPF should be able to determine the spatial and spectral distribution of zodiacal clouds with at least 0.1 times the brightness of the solar system's zodiacal cloud.

\section{Planet spectrum.}

Spectral Range. The required spectral range of TPF for characterization of extrasolar planets will emphasize the characterization of Earth-like planets and is therefore set to 0.5 to 0.8 micron in the visible and 6.5 to 13 microns in the infrared. The desired ranges are 0.5 to 1.05 micron and 6.5 to 17 microns.

Color. Colors distinguish planets from other objects. TPF must use color to characterize the type of planet and to measure its gross properties, including effective temperature at mid-IR wavelengths. Reference colors and relative magnitudes are those of Venus, Earth, Mars, and Jupiter. TPF must measure planet color in 3 or more bands, to an accuracy of $10 \%$, for any detected planet. We require that the ratio of color-characterized planets to all detected planets have an expectation value of at least $50 \%$.

Spectrum. TPF must use the spectrum of a planet to characterize its surface and atmosphere. The spectrum of the present Earth, scaled for semi-major axis and star luminosity, must be used as a reference. The required spectral resolution is 70 in the visible and 20 in the infrared. TPF must measure $\mathrm{O}_{2}, \mathrm{H}_{2} \mathrm{O}$, and $\mathrm{O}_{3}$ in the visible and $\mathrm{H}_{2} \mathrm{O}$ and $\mathrm{O}_{3}$ in the infrared. In this context, a measurement of a species is defined as the determination of the equivalent width of a spectral feature of that species to $20 \%$ accuracy. We desire that TPF measure Rayleigh scattering, photosynthetic pigments, $\mathrm{CO}_{2}$, and $\mathrm{CH}_{4}$ in the visible and $\mathrm{CO}_{2}$ as well as $\mathrm{CH}_{4}$ in the infrared. The desired spectral resolutions are 2 times the required values. See Section 3, below, for scientific importance of obtaining spectra of a planet in both the optical and mid-IR bands.

Number of Stars to Search. We require that TPF have the capability of searching at least 35 core stars, and additionally state the goal of being able to search at least 165 stars — being the total of 35 core stars plus 130 additional stars.

Extended Number of Stars. We desire to search as many stars as possible, beyond the required core and additional star groups. We anticipate that any mission capable of satisfying these objectives will also be capable of searching many more stars if the overall requirements are relaxed. Therefore we desire that TPF be capable of searching an extended group of stars defined as those systems of any type in which all or part of the continuously habitable zone (see below) can be searched.

Search Completeness. Search completeness is defined as that fraction of planets in the orbital phase space (defined above) that could be found within instrumental and mission constraints. We require each core star to be searched at the $90 \%$ completeness level. For further targets in addition to the core stars, the required $90 \%$ completeness is integrated over the additional stars.

Characterization completeness. It will be difficult to obtain spectra of the fainter or less well positioned planets. We require that the ratio of spectrally-characterized planets to all detected planets have an expectation value of at least $50 \%$.

Visitations. Multiple visits per star may be required to achieve completeness or to study a planet along its orbit, to determine its orbit, distinguish it from background objects, and validate a measurement. Therefore TPF must make enough visits to meet the completeness and other requirements. 
Multiple Planets. After the completion of the required number of visitations defined above, TPF should be able to characterize a planetary system as complex as our own with 3 terrestrial-sized planets assuming each planet is individually bright enough to be detected by TPF.

Orbit Determination. After the completion of the required number of visitations defined above, TPF shall be able to localize the position of a planet orbiting in the habitable zone with an accuracy of $10 \%$ of the semi-major axis of the planet's orbit. This accuracy may degrade to $25 \%$ in the presence of multiple planets.

\begin{tabular}{|c|c|c|}
\hline Key Parameter & Requirement & Goal \\
\hline Star types & F through $\mathrm{K}$ & $\mathrm{F}$ through $\mathrm{K}$ \\
\hline Habitable zone & 0.7 to $1.5 \mathrm{AU}$ scaled $\mathrm{L}^{0.5}$ & 0.7 to $1.5 \mathrm{AU}$ scaled $\mathrm{L}^{0.5}$ \\
\hline Orbit phase space & $\begin{array}{l}\text { Semi-major axis: uniform } \\
\text { inclination: uniform } \\
\text { eccentricity: } 0-0.35\end{array}$ & $\begin{array}{l}\text { Semi-major axis: uniform } \\
\text { inclination: uniform } \\
\text { eccentricity: } 0-0.35\end{array}$ \\
\hline Number of stars to be searched & 35 core stars & 165 additional stars \\
\hline Completeness per core star & $90 \%$ & $90 \%$ \\
\hline Completeness per set of additional stars & N/A & $\begin{array}{l}90 \% \text { integrated over the } \\
\text { ensemble }\end{array}$ \\
\hline Minimum planet area & 1/2 Earth area & 1/2 Earth area \\
\hline Geometric albedo & Earth & Earth \\
\hline Flux ratio & $\begin{array}{l}\text { At least } 3 \text { broad wavelength } \\
\text { bands; }\end{array}$ & $\begin{array}{l}\text { At least } 3 \text { broad wavelength } \\
\text { bands }\end{array}$ \\
\hline Spectral range & $\begin{array}{l}0.5-0.8[0.5-1.05] \mu \mathrm{m} \\
6.5-13[6.5-17] \mu \mathrm{m}\end{array}$ & $\begin{array}{l}0.5-0.8[0.5-1.05] \mu \mathrm{m} \\
6.5-13[6.5-17] \mu \mathrm{m}\end{array}$ \\
\hline Characterization completeness & $50 \%$ & $50 \%$ \\
\hline Giant planets & $\begin{array}{l}\text { Jupiter brightness at } 5 \mathrm{AU}, \\
50 \% \text { of stars }\end{array}$ & $\begin{array}{l}\text { Jupiter brightness at } 5 \mathrm{AU}, \\
50 \% \text { of stars }\end{array}$ \\
\hline Average (Maximum) tolerable exozodi & 3 (10) zodi & 3 (10) zodi \\
\hline
\end{tabular}

Table 1. Summary of TPF science objectives

\subsection{Summary}

Table 1 summarizes the objectives for TPF. Note that the terms "requirements,, and "goals,, are used loosely at this phase of the project. Formal definitions will be set by NASA Headquarters later in the definition of the project.

As a minimum, TPF must be able to detect planets with half the area of the Earth, and the Earth's geometric albedo, searching the entire $\mathrm{HZ}$ of the core-group stars with $90 \%$ completeness per star. Flux ratios must be measured in 3 broad wavelength bands, to $10 \%$ accuracy, for at least $50 \%$ of the detected terrestrial planets. The spectrum must be measured - for at least $50 \%$ of the detected terrestrial planets - to give the equivalent widths of $\mathrm{O}_{2}, \mathrm{H}_{2} \mathrm{O}$, and $\mathrm{O}_{3}$ in the visible or $\mathrm{H}_{2} \mathrm{O}$, and $\mathrm{O}_{3}$ in the infrared to an accuracy of $20 \%$. 
As a goal, TPF must be able to detect planets with half the area of the Earth, with Earth's geometric albedo, searching the entire $\mathrm{HZ}$ of the 35 core-group stars plus the aggregated HZs of at least 130 additional stars with $90 \%$ completeness for both groups. Flux ratio must be measured in 3 broad wavelength bands to $10 \%$ accuracy for at least $50 \%$ of the detected terrestrial planets. The spectrum must be measured-for at least $50 \%$ of the detected terrestrial planets-to give the equivalent widths of $\mathrm{O}_{2}, \mathrm{H}_{2} \mathrm{O}$, and $\mathrm{O}_{3}$ in the visible or $\mathrm{H}_{2} \mathrm{O}$, and $\mathrm{O}_{3}$ in the infrared to an accuracy of $20 \%$. Further, we desire that the mission search an extended group of stars defined as those systems of any type in which all or part of the HZ can be searched.

\section{OPTICAL AND MID-INFRARED SPECTROSCOPY}

As described above, NASA is currently pursuing two mission designs for TPF - a coronagraph operating in the visible waveband, and a free-flying interferometer operating in the mid-infrared. If one were able to perform spectroscopy of an Earth-like planet in either the optical or the mid-IR wavebands, one might pose the question: which is the preferable waveband from a scientific viewpoint? Which waveband offers the best opportunity to characterize the atmosphere of a planet and infer its physical and chemical properties, and its suitability for harboring life?

The astrobiology community has repeatedly emphasized the complementary nature of data in the two wavelength ranges and has urged that in the long run, data at both wavelengths are required to understand the physical conditions on the planet's TPF detects, to characterize planetary atmospheres, and to carry out a robust search for life. We must bear in mind that there exists a huge possible range of physical and chemical conditions in extrasolar planets. The planets in our own Solar System provide a guide, but they necessarily represent a (possibly small) subset of possible planetary surface and atmosphere conditions that TPF may encounter.

Perhaps the most definitive statement on this question is contained in the report by Des Marais et al. ${ }^{11}$, in which they explore the signatures of many atmospheric components under a variety of physical conditions: "Both the mid-infrared and the visible to near infrared spectral ranges offer valuable information regarding biomarkers and planetary properties, therefore both ranges merit serious scientific consideration for TPF. The best overall strategy for the Origins program includes a diversity of approaches, therefore both wavelength ranges ultimately should be examined prior to launching the "Life-Finder,, mission.,"

\subsection{Physical Properties}

For decades, planetary astronomers have relied on the combination of thermal and visible light observations of solar system objects to understand the most basic properties of these objects. For example, determining the size of a spatially unresolved asteroid or minor planet requires observation of both thermal emission (proportional to size and temperature) at IR wavelengths and reflected sunlight (proportional to size and reflectivity) at visible wavelengths. With observations from both wavelength regions it is possible to distinguish a shiny, small planet from the darker, larger one; the two would have similar visual appearance but very different infrared properties. Alternatively, two significantly different planets (e.g., a small hot planet and a larger cooler planet) could look the same at mid-IR wavelengths but very different at visible wavelengths.

Similar arguments apply to planets in other solar systems. Determining the size, temperature, albedo, and thus the global energy balance of a planet is critical to assessing a planet's habitability. For example, the planet's temperature tells us the likelihood of liquid water existing on its surface. While temperature is derived from mid-IR wavelengths (with temperature, albedo, and size best separated with multi-wavelength observations), visible wavelength observations can help tell us if the temperature is actually that measured at the surface. Multi-wavelength observations are further useful because there may be many planets whose brightness is such that spectroscopy is impossible in first generation missions. Comparison of low resolution, visible and mid-IR flux densities would allow us to understand these planets well in advance of a more capable spectroscopic mission. 


\subsection{Atmospheric Properties}

Figure $1\left(\mathrm{Traub}^{12}\right)$ shows a number of spectral lines that might be found in common between the visible and near-IR spectra from TPF. The ability to study multiple lines provides confirmation of initial detections and greatly extends the possibilities of interpretation. For example, the mid-IR $\mathrm{CO}_{2}$ absorption line is saturated. While this line will be easy to detect at very low concentrations, at high concentrations the $\mathrm{CO}_{2}$ abundance may be hard to infer. A simultaneous solution for the strengths of mid- and near-IR lines (1-2 $\mu \mathrm{m})$ of $\mathrm{CO}_{2}$ would allow one to extract information on concentration, temperature, vertical temperature gradient, and atmospheric pressure that would be hard to obtain from a single line. Comparable arguments could be made for other species where unraveling the effects of optical depth, the presence of clouds, and vertical temperature gradients demand a great deal of information for accurate modeling.

A second area of complementarity comes from the fact that while IR observations may not provide much insight into the surface properties of a planet with a substantial atmosphere, visible light can provide information on the surface conditions if the planet is not fully covered with clouds. Such information might include identification of liquid water oceans, continents, or biological pigments indicative of land plants. If the planet is covered in clouds, then many of the atmospheric signatures will depend on the altitude and thickness of the clouds for both the visible and the IR and combined data will again prove useful.

\subsection{Confirming the Presence of Life}

The most unambiguous biomarker in a planet's atmosphere is the simultaneous presence of significant concentrations of free oxygen along with a reduced gas such as $\mathrm{CH}_{4}$ or $\mathrm{N}_{2} \mathrm{O}$. The next best bioindicator is free oxygen itself. There are great benefits in searching for oxygen at two different wavelengths. The strength of the $\mathrm{O}_{3}$ band of ozone around 9.6 $\mu \mathrm{m}$ has a weak, nonlinear dependence on $\mathrm{O}_{2}$ concentration, making this band a critical proxy for $\mathrm{O}_{2}$ over many orders of magnitude variation in $\mathrm{O}_{2}$ abundance. This is an important attribute since the $\mathrm{O}_{2}$ abundance is expected to vary widely as photosynthesis evolves on a planet. On the other hand, the $\mathrm{O}_{3}$ line is relatively insensitive to the actual $\mathrm{O}_{2}$ abundance as long as it is above $\sim 1$ percent of the present level. Thus, the detection of both $\mathrm{O}_{3}$ and $\mathrm{O}_{2}$ yields both a critical confirmation of the presence of oxygen in planet's atmosphere - the smoking gun for the presence of life---as well as more information on the amount of this gas and its distribution in the atmosphere.

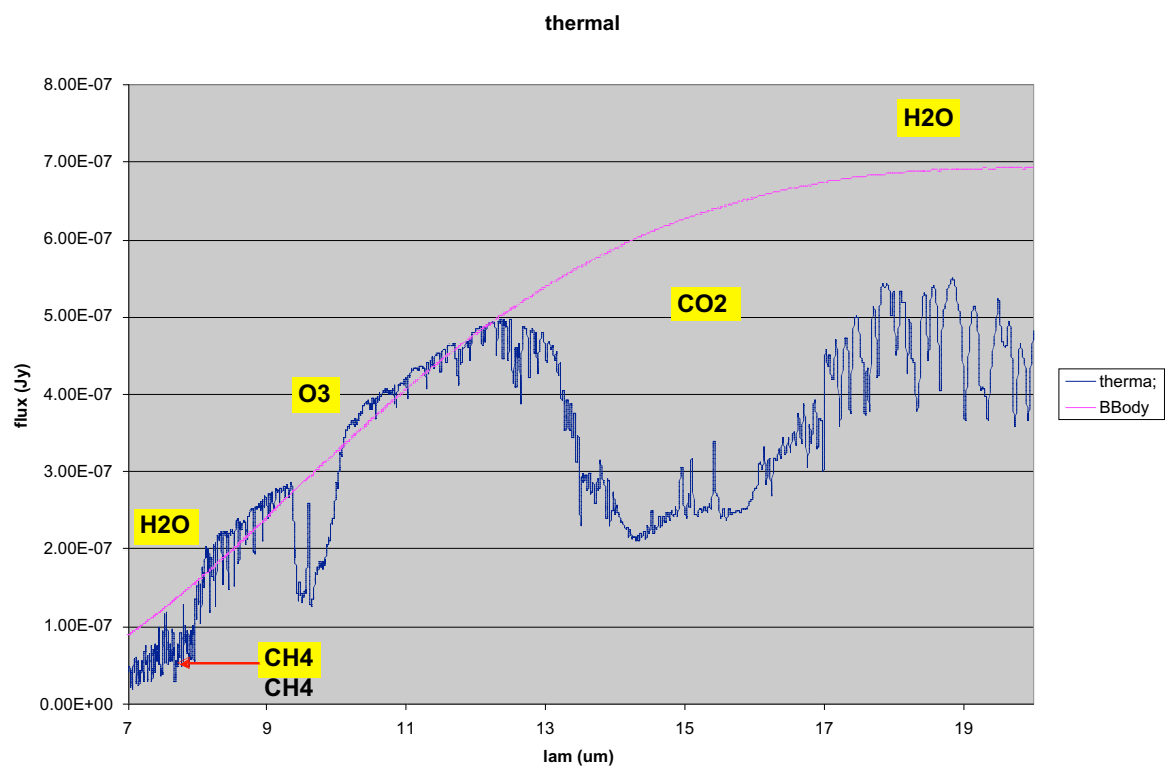

Having data in two different wavelength regions would also help with the identification of the secondary biomarker gases $\mathrm{CH}_{4}$ and $\mathrm{N}_{2} \mathrm{O}$. Both of these gases are derived primarily from biological activity on Earth today. They would be difficult to detect from interstellar distances in Earth's present atmosphere because they have relatively low concentrations (1.7 ppmv for $\mathrm{CH}_{4}, 0.3$ ppmv for $\mathrm{N}_{2} \mathrm{O}$ ). However, they may be more prominent in $\mathrm{O}_{2}$-rich atmospheres 
of planets around late $\mathrm{K}$ and $\mathrm{M}$ stars (because of lower far-UV fluxes), and $\mathrm{CH}_{4}$ is expected to be present at high concentrations (1000 ppmv or more) in anoxic, post-biological, early Earth-type atmospheres. $\mathrm{CH}_{4}$ can be observed in both the visible and the thermal infrared, whereas $\mathrm{N}_{2} \mathrm{O}$ can only be seen in the latter wavelength region. Unfortunately, the observable thermal-IR bands of $\mathrm{CH}_{4}$ and $\mathrm{N}_{2} \mathrm{O}$ are both centered near $7.7 \mu \mathrm{m}$, so it would be difficult to distinguish between them based on thermal-IR data alone. Thus, detecting both of these gases unambiguously would require data in both wavelength regions. It is very clear to the spectroscopists and atmospheric chemists who have thought about the problem that if we make a tentative identification of life based on data from one wavelength region, data from the other wavelength region will be critical to confirming that conclusion.

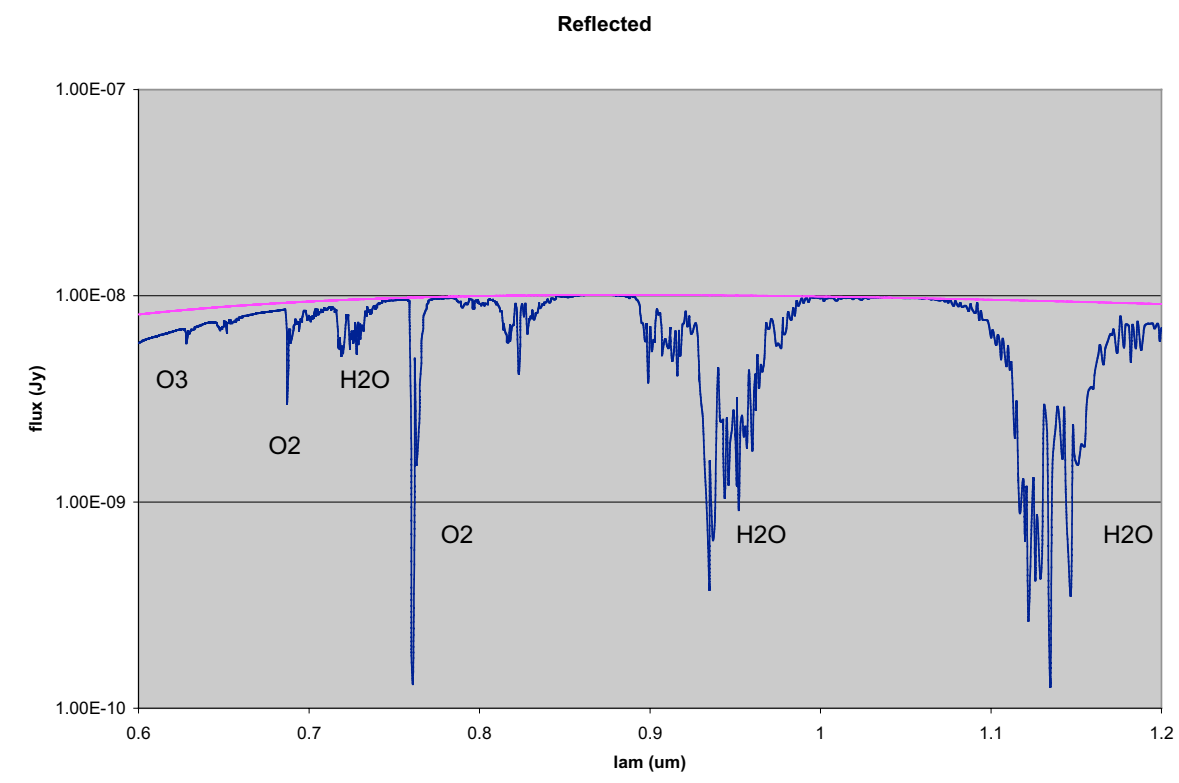

\subsection{Non-Simultaneous Observations}

The power of both mid-IR and visible observations would be, of course, be maximized if the observations could be taken contemporaneously. But even if the data were obtained 5-10 years apart, it would be possible to combine the datasets in a meaningful manner given the relatively slow rate of change of the gross physical properties being considered here. Account would have to be taken of orbital location, phase-function effects, etc., but with numerous observations at both wavelengths it would be possible to model these effects and take full advantage of the data.

\section{GENERAL ASTROPHYSICS}

An observatory with the power to detect a $30^{\text {th }}$ mag object in the visible a few tenths of an arcsecond away from a $5^{\text {th }}$ mag star, or of taking a spectrum of a $0.3 \mu \mathrm{Jy}$ planet at $10 \mu \mathrm{m}$, must be a formidably powerful machine, capable of many other exciting observations. Even so, the ability to perform general astrophysics observations will in no way be allowed to impair the capability of TPF for planet finding or of taking observing time away in TPF's primary goal by focusing too much on these other projects. The TPF project has divided these projects, distinct from the search for habitable planets, into a number of categories (Figure 2):

1. A program of comparative planetology is a fundamental part of the primary mission of TPF, i.e. to understand the environment of habitable planets including gas giant planets and residual clouds of gas and dust. Exterior gas giants may protect interior planets from excessive bombardment. Populations of comets or asteroids may provide the volatiles needed for life. Understanding all these constituents of solar systems is necessary for a complete understanding of the formation and evolution of habitable planets and life. 
2. General astrophysical observations are possible with existing TPF instrumentation with little or no modification. Such observations include probing the central arcsecond of important classes of objects such as protostars, mass-losing evolved stars, the cores of our own and other galaxies, distant quasars, and galaxies forming at high redshifts. TPF data obtained with high sensitivity, high angular resolution, and high contrast will uniquely complement data obtained with other facilities such as SIM, the James Webb Space Telescope (JWST), and the ground-based Atacama large Millimeter Array (ALMA).

3. Some extended astrophysical observations require either new instrumentation or significant modifications to the facility but would offer tremendous potential for breakthrough science. Examples include milli-arcsecond imaging of protostars at 3-10 $\mu \mathrm{m}$ with interferometric baselines as long as $300 \mathrm{~m}$, or deep, wide field (5 arcminute) searches for supernovae in the visible/near-IR with 10-20 milli-arcsecond resolution. The addition of such capabilities will be limited by the availability of funding, observing time, and the criticality of the observations in light of other space-based and ground-based facilities.

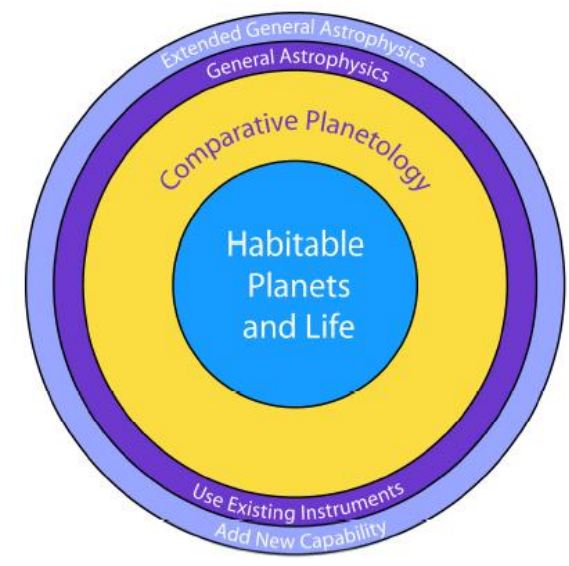

Figure 2. A schematic representation of the TPF capabilities and science programs.

\section{ACKNOWLEDGMENTS}

The research described in this paper was carried out by the Jet Propulsion Laboratory, California Institute of Technology, under a contract with the National Aeronautics and Space Administration. The science objectives described here were developed as a collaboration of many scientists interested in the search for Earth-like planets and the study of extrasolar planetary systems. In particular, we thank the members of the TPF Science Working Group for their contributions, especially to the draft science objectives presented here.

\section{REFERENCES}

1. R. A. Laskin, “SIM technology development overview,,, Proc SPIE, 4852, 16, 2003

2. M. Mayor \& D. Queloz, “A Jupiter-Mass Companion to a Solar-Type Star,,, Nature, 378, 355, 1995.

3. G. Marcy, R. P. Butler, “A Planetary Companion to 70 Virginis,„, ApJ, 464, L147, 1996.

4. S. Ida, D. N. C. Lin, "Toward a Deterministic Model of Planetary Formation. I. A Desert in the Mass and Semimajor Axis Distributions of Extrasolar Planets,,, ApJ, 604, 388, 2004.

5. M. M. Colavita, P. L. Wizinowich, "Keck Interferometer update,,, Proc SPIE, 4838, 79, 2003.

6. G. T. van Belle, A. F. Boden, M. M. Colavita, M. Shao, G. Vasisht, J. K. Wallace, "Astrometry with the Keck Interferometer ,„, Proc SPIE, 3350, 362., 1998

7. S. C. Unwin, "SIM Science Operations," Proc SPIE, 4852, 172, 2003. 
8. C. Snodgrass, K. Horne, Y. Tsapras, "The abundance of galactic planets from OGLE-III 2002 microlensing data,,, Mon. Not. R. Astron. Soc. (in press), 2004.

9. Bond, et al., "OGLE 2003-BLG-235/MOA 2003-BLG-53: A Planetary Microlensing Event,,, ApJ, 606, 155, 2004.

10. W. J. Borucki, et al., "The Kepler mission: a wide-field-of-view photometer designed to determine the frequency of Earth-size planets around solar-like stars,,, Proc SPIE, 4854, 129, 2003

11. D. J. Des Marais, M. O. Harwit, K. W. Jucks, J. F. Kasting, D. N. C. Lin, J. I. Lunine, J. Schneider, S. Seager, W. A. Traub, N. J. Woolf, "Remote Sensing of Planetary Properties and Biosignatures on Extrasolar Terrestrial Planets,,, Astrobiology, 2, 153, 2002.

12. W. Traub (private communication), 2004. 